connection with transformation groups. And besides these reviews by German mathematicians there is an account by Professor Eddy, now of the University of Minnesota, of the remarkable development of graphical methods within the past thirty years, particularly in connection with engineering theory and practice.

The same general interest attaches to the paper in which Professor Hilbert, now of Göttingen, gives an outline of his own important investigations in the field of algebraic invariants; to that of Professor Minkowski, now of Königsberg, on his geometry of integral numbers; of Professor Pringsheim, of Munich, on his general theory of the divergency and convergency of series with positive terms; of Professor Study on his researches in spherical trigonometry; and of Mr. D'Ocagne, of Paris, on his powerful graphical method: "Nomographie."

But the volume is not simply a collection of expository papers and resumés. It contains a full share of valuable and interesting original papers also. In the field of the theory of substitutions and algebraic equations are papers by Cole now of Columbia, Maschke and Moore of Chicago, de Perrott and Taber of Clarke and Weber of Strasburg; in the field of functions, papers by Bolza of Chicago, Hermite of Paris, Krause of Dresden, Pincherle of Bologna and Stringham of the University of California; while mathematical history, the reduction of binary quantics, continuous transformation groups, algebraic curves, the Grassmann analysis and geodesic lines are represented by papers by Halsted of the University of Texas, Hurwitz of Zürich, Meyer of Clausthal, Noether of Erlangen, Schlegel of Hagen and Weyr of Prague respectively. From every point of view, therefore, this volume is an important contribution to the mathematical literature of America. It is, moreover, carefully edited and beautifully printed.

Henry B. Fine.

\title{
ELECTRICITY AND MAGNETISM.
}

Elements of the Mathematical Theory of Electricity and Magnetism. By J. J. Thоmson, F. R. S., Cavendish Professor of Experimental Physics in the University of Cambridge. vi+ 510 pp., octavo. Cambridge, University Press; New York, The Macmillan Company, 1895. \$2.60.

When Professor Thomson undertook to edit a new edition of Clark Maxwell's treatise it was hoped he would re- 
model parts of it in order to make clearer some conceptions upon which subsequent thought and experiment have thrown new light, and remove certain inconsistencies in Maxwell's method of presentation. It was felt that Professor Thomson was just the man to do this, and after consideration of the volume before us we are quite certain of it. Accordingly there was some disappointment when few changes were made, and the additions were collected in an extra volume, and not incorporated into the text. We presume that it was considered wiser not to tamper with Maxwell's classic treatise. When the publishers announced an elementary text-book on the mathematical theory of electricity and magnetism by J. J. Thomson, we were led to expect something new and individual in treatment, and embodying the latest work on the subject; this expectation has been fully realized. There was no dearth of text-books on this subject before, but they were, one and all, very unsatisfactory; although written presumably for physicists, the physical aspect of the subject was frequently not even considered. Perhaps the first impression after reading a few dozens of pages at the beginning of the volume before us, is that there is too close an adherence to Maxwell's matter and method ; but the individuality of the book is soon apparent, new methods of presentation please us, and difficult theorems yield before a simple treatment and careful choice of illustrative cases.

The first five chapters are devoted to the consideration of electro-statics. The author, at an early stage, introduces the use of Faraday tubes. Their value in the formation of mental pictures of the phenomena, not only of electrostatics, but of the whole subject, he conclusively shows us in an excellent chapter in his "Recent Researches." To the student of physics, in general, and of electricity, in particular, all such aids to thought are especially acceptable. The more use is made of the notion of potential, as work done, and of lines of force in the explanation of such phenomena as the variation of capacity, the attractions and repulsions of electrified bodies, the energy in the various parts of the field, etc., the more hold has the student upon the theorems considered. The last few sections of Chapter II. of this book, and again Article 74, afford an admirable example of the uses to which the conception of tubes of force may be put.

There is one point about the book which seems to us to spoil somewhat its excellence; that is, a certain amount of what has been called "calculus dodging." It is hard to 
see why a theorem such, for example, as Gauss's, Art.10, or the energy in the electric field, Art. 23, should be proved by such long round-about geometrical or other methods, rather than by the use of the shorter calculus; especially as calculus is used in all parts of the book.

The author states in his preface that very often important theorems are left out of elementary text-books, because their proof in their most general form demands higher analysis than can be required of the student, and contends " that often the study of the cases which will yield to very simple mathematical treatment fully illustrates the physical phenomena, and gives the student a more vivid idea and a more manageable grasp of the subject than he would be likely to attain, if he merely regarded electrical phenomena through a cloud of analytical symbols." A confirmation of this is his treatment of the law of the inverse square in attraction, Art. 20, and his method of finding the tensions and pressures in the ether. The latter is a case of other than calculus methods referred to above which is eminently proper. Again see Articles 231 and 239 on the distribution of currents in a conductor.

It is interesting to see the whole subject of dielectrics considered without the introduction of that unnecessary phrase "fictive layer." If we are to follow Maxwell's theory, what real difference is there between a charge and a "fictive layer" of electricity?

Chapters VI., VII. and VIII. are devoted to magnetism. The student of electrical engineering will regret that more space is not given to a discussion of magnetic circuits, reluctance, etc.

Then follow chapters on electric currents, electromagnetism, units, dielectric currents and a very short chapter on thermo-electric currents. A notable novelty is his treatment of the actual distribution of currents in a network of wires by introducing the dissipative function, that is, the expression for the rate of heat production. He shows that it is only necessary to write down this expression and make it a minimum, subject to the condition that the algebraical sum of the currents which meet in a point is zero, in order to find these currents. The discussion of electrolysis is exceedingly scanty.

In a volume that is so generally excellent, it is not easy to point out parts of special merit, but perhaps the chapters on specific inductive capacity and electro-magnetic induction are particularly noteworthy. The usefulness of the book would be increased greatly by more complete tables, 
and by copious references : in the latter point it is entirely deficient. The freedom from mistakes is worthy of note in a first edition, if we neglect the inaccuracies in the reference to articles. In the last line of p. 144 the last term should be $\frac{a}{f^{2}-a^{2}}$ not $\frac{e a}{f^{2}-a^{2}}$. In line 8 of p. 157 the last term should be $\frac{1}{2} \frac{b^{2}}{a^{2}}$ not $\frac{1}{2} \frac{b^{2}}{a^{3}}$.

A. Stanley Mackenzie.

Bryn Mawr College, Bryn MaWr, Pa.

\section{THE SUBSTITUTION GROUPS WHOSE ORDER IS THE PRODUCT OF TWO UNEQUAL PRIME NUMBERS.}

BY DR. G. A. MILLER.

The regular groups of order $p q$ ( $p$ and $q$ represent prime numbers, $p>q$ ) were determined by Netto.* It is the object of this paper to determine all the substitution groups of this order and to find formulas by means of which their number may be readily found for any given values of $p, q$ and $n \dagger$ even if the groups themselves are unknown.

We shall determine the cyclical and the noncyclical groups separately. It will be found that the number of the noncyclical groups, when the values of $p$ and $q$ are such that groups of this type occur, is always equal to or one larger than the number of the cyclical groups for any given values of $p, q$ and $n$.

We shall employ the symbol $R G_{p q}^{m p q}$ to represent the intransitive group which is obtained by establishing a simple isomorphism between $m$ regular groups of order $p q$. The symbol $G_{y}^{x}$ will be used to represent, as usual, any group whose degree is $x$ and whose order is $y$.

\section{$\S 1$ \\ The Cyclical Groups.}

Each one of these groups belongs to one and only one or the following three classes :

\footnotetext{
* Theory of Substitutions (Cole's edition) \& 130.

$+n$ represents the degree of the groups.
} 